\title{
Chemoembolization and stenting combined with iodine-125 seed strands for the treatment of hepatocellular carcinoma with inferior vena cava obstruction
}

\author{
WENHUI LI ${ }^{1}$, ZHENYU DAI ${ }^{1}$, LIZHENG YAO ${ }^{1}$, JIANJUN LUO ${ }^{2}$ and ZHIPING YAN ${ }^{2}$ \\ ${ }^{1}$ Department of Interventional Radiology, Yancheng Hospital, Dongnan University, Yancheng, Jiangsu 224001; \\ ${ }^{2}$ Department of Interventional Radiology, Zhongshan Hospital, Fudan University, Shanghai 200032, P.R. China
}

Received August 4, 2014; Accepted April 13, 2015

DOI: $10.3892 /$ etm.2015.2581

\begin{abstract}
The aim of the present study was to investigate the efficacy and safety of stenting combined with radioactive iodine-125 seed strands following chemoembolization for the treatment of patients with hepatocellular carcinoma and inferior vena cava (IVC) obstruction. A retrospective analysis was conducted of 52 hepatocellular carcinoma patients with IVC obstruction. All patients received chemoembolization of tumor-supplying arteries and IVC stents, and 18 patients additionally received iodine-125 seed strands, which were fixed to the stents. Improvement of IVC obstruction and the tumor response rates were compared between the two groups with a median follow-up time of 2.5 months. In both groups the stents were successfully deployed. At the 2-month post-procedural follow-up, the mean diameter of the IVC obstruction site, the mean pressure difference between the distal IVC obstructive segment and the right atrium as well as the obstruction scoring did not differ significantly between the two groups. By contrast, the tumor response rate of the iodine-125 seed strand group was $94.4 \%$, whereas for the group without iodine-125 seed strands it was $35.3 \%(\mathrm{P}<0.001)$. The combination of stent and iodine-125 seed strands was effective and safe for the treatment of hepatocellular carcinoma with IVC obstruction.
\end{abstract}

\section{Introduction}

Hepatocellular carcinoma (HCC) is the sixth common cancer and the third most cause of cancer-related mortality in the world $(1,2)$ with a high incidence of complications. Between 13 and $23 \%$ of HCC patients suffer inferior vena cava (IVC) obstructions caused by tumor metastasis and/or lymph node

Correspondence to: Professor Jianjun Luo, Department of Interventional Radiology, Zhongshan Hospital, Fudan University, 180 Fenglin Road, Shanghai 200032, P.R. China

E-mail: luojianjunbm@163.com

Key words: inferior vena cava obstruction, radioactive iodine-125, stent, hepatocellular carcinoma invasions (3), which is frequently associated with lower extremity edema, ascites, renal dysfunction and even fatal pulmonary infarction due to cancer thrombus shedding. The average survival time of HCC patients with IVC obstruction has been reported to be only 2-3 months without effective treatment (4). At present, various options are available for the treatment of HCC with IVC obstruction, including surgery, transcatheter arterial chemoembolization (TACE), IVC stent placement, radiotherapy and radioactive iodine-125 seeds. Surgical resection is often restricted when the tumor is in advanced stage with poor liver function and tumor invasion of vessels and bile ducts. The efficacy of TACE for HCC has been widely accepted and the treatment can significantly relieve obstructions of the IVC, improve liver function and the quality of life for a short period when combined with stent implantation; however, the rate of stent restenosis is high with poor long-term outcomes (5). The main characteristics of iodine-125 are constant low energy X-ray and $\gamma$-ray release, which inhibit or kill tumor cells in a maximum radius of only $17 \mathrm{~mm}$ with little damage to the surrounding tissues. It has also been reported that iodine-125 is able to inhibit vascular intimal hyperplasia, thus reducing the incidence of stent restenosis $(6,7)$. Brachytherapy with iodine-125 has been widely used for the treatment of prostate cancer and head and neck tumors as well as liver cancers, mainly by implanting iodine-125 seeds into the tumor sites (8). The present study is a retrospective analysis of combined treatment with iodine-125 seed strands with TACE and stent treatments for IVC obstructions in patients with $\mathrm{HCC}$ in comparison with conventional TACE and stent treatments.

\section{Patients and methods}

Patients. This study has been approved by the hospital ethics committee (Yancheng Hospital, Dongnan University, Yancheng, Jiangsu, China) and written informed consent was obtained from all participants. From March 2009 to June 2013, 52 patients with HCC and IVC obstruction were treated in the hospital and retrospectively analyzed. Inclusion criteria were: i) no history of IVC obstruction treatments, ii) symptoms clearly associated with IVC obstruction, iii) Child-Pugh score $\mathrm{A}$ or $\mathrm{B}$, with an expected survival time of $>3$ months. 
Exclusion criteria were: i) pulmonary metastasis; ii) tumor thrombus of the right atrium; iii) right cardiac insufficiency. The symptoms associated with IVC obstruction were scored with 1 point for mild lower limb edema, 2 points for severe edema, 1 point for abdominal wall superficial varicose veins and 1 point for scrotal edema. All patients underwent pre-procedural hepatic and renal function tests, blood routine and contrast-enhanced abdominal computed tomography (CT) examinations to assess liver lesions and lymph node metastasis as well as types (compression, endovascular invasion or tumor thrombus) of IVC obstructions.

\section{Surgical technique}

Main instruments. The stenting instruments were a Z-type metal self-expandable venous stent (diameter $30 \mathrm{~mm}$ and length $100 \mathrm{~mm}$; Youyan Yijin, Beijing, China) and iodine-125 seeds (type 6711, length $4.5 \mathrm{~mm}$, radioactivity $0.7 \mathrm{mCi} / \mathrm{seed}$, effective radiation radius $17 \mathrm{~mm}$; Ningbo Junan Pharmaceutical Technology Company, Ningbo, China).

Implantation of stent and iodine-125 seed strands. Following local anesthesia with the patient in a supine position and electrocardiogram (ECG) monitoring, the right femoral vein was punctured and a 4-F pigtail catheter was inserted for IVC venography. This was to obtain diameter and length measurements for the obstructed segment of the IVC, to determine the pressure difference between the distal IVC obstructed segment and the right atrium, and to observe the collateral circulation. According to pre-stenting CT findings and IVC venography, stents and iodine-125 seed strands were prepared for transplantation (stent and seed strands arranged in line were required to cover and exceed $10 \mathrm{~cm}$ on either end of the obstructed segment of the IVC). For patients with IVC stenosis (tumor invasion or external pressure) $<50 \%$, only a single seed strand was implanted; for patients with IVC stenosis $>50 \%$, two seed strands were used to ensure treatment efficacy, since the effective radius of iodine- 125 is only $17 \mathrm{~mm}$. The required amount of iodine-125 seeds was packaged in sterile medical plastic 4-F tubes in a line to form iodine-125 seed strands, which were fixed to the stent surface by surgical suture. For implantation of single seed strands, a 0.035 -inch guide wire was first inserted through the obstructed segment followed by a $12-\mathrm{F}$ long sheath along the guide wire with the sheath exceeding the proximal IVC obstructive segment by $10 \mathrm{~mm}$. The stent with iodine-125 seed strands was then carried into the 12-F long sheath with an importer under posteroanterior and lateral X-ray fluoroscopy, pushed to the long sheath head end and rotated along the sheath so that the iodine-125 seed strand lay close to the lesion side of the IVC. Finally the long sheath was retreated and the stent released (Fig. 1). For implantation of two iodine-125 seed strands, after a $12-\mathrm{F}$ long sheath with stent and single seed strands was positioned in the discharge position via a right femoral vein approach, the right jugular vein was punctured, a 6-F sheath was inserted into the IVC and the second iodine-125 seed strand was delivered to the targeted position. The 12-F long sheath was then retreated and released $\sim 2 / 3$ of the length of the stent. Then, the $12-\mathrm{F}$ long sheath was rotated and the position of the seed strands in the 6-F sheath was adjusted by the force of the stent in the 12-F sheath under posteroanterior and lateral X-ray fluoroscopy, to result in two seed strands lying $\sim 1.5 \mathrm{~cm}$ apart and close to
A

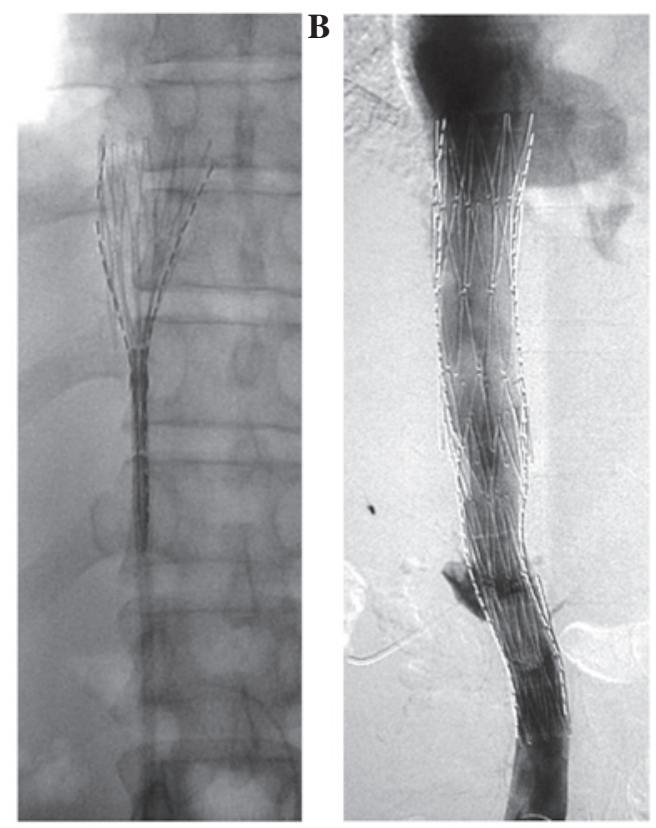

Figure 1. (A) Retreated sheath and 2/3 release of the stent. (B) Completely released stent and opened stenotic segment of the inferior vena cava.
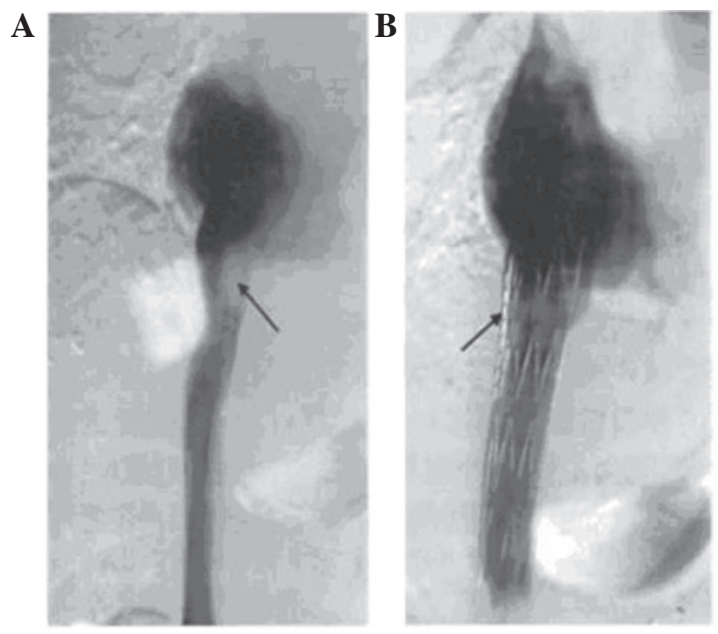

Figure 2. (A) Pre-stenting venography showing the stenotic segment of the IVC (arrow). (B) Post-stenting venography showing the opened stenotic segment of the IVC (arrow). IVC, inferior vena cava.

the obstructed IVC segments. The 12-F sheath was retreated and the stent completely released. Finally, the 6-F sheath was retreated and the other seed strands were placed between the stent and the vessel wall of the IVC. IVC venography was performed again (Fig. 2) and the treatment was complete if venography showed that the IVC blood flow patency was good, the collateral veins disappeared or decreased significantly, and the diameter of the obstructed segment of the IVC segment and the pressure difference between the distal obstructed IVC segment and the right atrium after stent implantation were acceptable. If not, a balloon of diameter $20 \mathrm{~mm}$ and length $40 \mathrm{~mm}$ was inserted to dilate the stent in order to achieve angiographic images meeting the above criteria.

TACE technique. After right femoral artery puncture, a 4-F glide catheter was pushed to the coeliac artery for arteriography, and then into the superior mesenteric artery, left 
Table I. Comparison of basic characteristics between groups with and without iodine-125 seed strand implantation.

\begin{tabular}{|c|c|c|}
\hline Variable & With iodine-125 $(n=18)$ & Without iodine-125 $(n=34)$ \\
\hline Number of patients & 18 & 34 \\
\hline \multicolumn{3}{|l|}{ Gender, $\mathrm{n}$} \\
\hline Male & 18 & 27 \\
\hline Female & 0 & 7 \\
\hline Average age, years & $61 \pm 10$ & $62 \pm 10$ \\
\hline \multicolumn{3}{|l|}{ Child-Pugh grade } \\
\hline A & 15 & 28 \\
\hline $\mathrm{B}$ & 3 & 6 \\
\hline Serum AFP $\geq 400$ ng/ml, n & 18 & 34 \\
\hline Location of tumor & Hepatic hilar & Hepatic hilar \\
\hline Score of IVC obstructive symptom & $2-3$ & $2-3$ \\
\hline Position of IVC obstruction & Hepatic segment & Hepatic segment \\
\hline \multicolumn{3}{|l|}{ Type of IVC obstruction, $n$} \\
\hline Compression & 1 & 4 \\
\hline Invasive & 17 & 27 \\
\hline Tumor thrombus & & 3 \\
\hline Average diameter of IVC obstruction, mm & $5 \pm 2$ & $5 \pm 2$ \\
\hline $\begin{array}{l}\text { Average pressure difference between the distal site } \\
\text { of IVC obstruction and the right atrium, } \mathrm{mmHg}\end{array}$ & $17.4 \pm 2$ & $17.3 \pm 2$ \\
\hline
\end{tabular}

The basic characteristics did not show significant differences between the groups ( $\mathrm{P}>0.05)$. AFP, $\alpha$-fetoprotein; IVC, inferior vena cava .

gastric artery and bilateral phrenic artery for angiography when necessary, in order to determine the tumor-supplying artery. Next, another catheter was pushed to the target vessel (a 3-F micro-catheter was used when selection was difficult). When the catheter was in position, 0.5-1 g 5-fluorouracil and 100-200 mg oxaliplatin plus 10-60 mg lyophilized pirarubicin added to 5-20 ml Lipiodol was infused for embolization of the tumor-supplying artery according to the CT findings prior to the procedure, and the liver function and angiography results of the patients.

Post-procedural observation and follow-up. After stenting, all patients received an intravenous injection of $20 \mathrm{mg}$ furosemide to prevent heart failure and symptomatic anti-inflammatory and hepatoprotective treatments under ECG monitoring. At 1 day after stenting, all patients underwent single-photon emission computed tomography (SPECT)/CT examinations to examine the stent implantation and position of iodine-125 seed strands and observe the radiation distribution of implanted iodine-125. At 3 days after stenting, symptoms associated with IVC obstruction were re-scored. Patients were followed-up monthly in the outpatient department, and liver and kidney functions, blood routine examination as well as upper abdominal CT examinations were conducted to monitor the IVC patencies and changes of hepatic lesions after treatment. The tumor mass reduction rate was judged by response evaluation criteria in solid tumors (RECIST) criteria (http://www. irrecist.com/recist/): i) complete response (CR) was complete disappearance of the tumor; ii) partial response (PR) was shrinkage of the tumor size by $\geq 30 \%$; iii) stable disease (SD) was shrinkage of the tumor size by $<30 \%$ or an increase of tumor size of $<20 \%$; iv) progression of disease (PD) was an increase of tumor size by $\geq 20 \%$ or the appearance of new lesions. When existing residual lesions or new lesions were diagnosed, TACE was performed again 6-8 weeks after the last treatment.

Statistical analysis. The data are expressed as mean \pm standard deviation. Basic data of the groups, pre- and post-stenting obstruction indices in the two groups and indices of obstruction between two groups following the procedure were compared with t-tests. The tumor response rates between the two groups were compared by $\chi^{2}$ test. SPSS software, version 13.0 (SPSS, Inc., Chicago, IL, USA) was used for data processing, and $\mathrm{P}<0.05$ was considered to indicate a significant difference.

\section{Results}

Basic characteristics of the patients. Based on the inclusion criteria, 108 patients with HCC and IVC obstruction were selected and 18 of them accepted TACE and stents combined with iodine-125 seed strands. From the remaining 90 patients who accepted TACE and stent treatments but rejected iodine-125 seeds, 34 subjects with no significant differences of basic characteristics compared with the above 18 subjects were selected; thus, 52 patients (34 with TACE and stent as well as 18 with TACE, stent and iodine-125 seed treatments) were involved in this study (Table I). The average age was 
Table II. Comparison of IVC obstruction with and without iodine-125 seed strands.

\begin{tabular}{|c|c|c|c|c|}
\hline \multirow[b]{2}{*}{ Variable } & \multicolumn{2}{|c|}{ With iodine-125 seed strands } & \multicolumn{2}{|c|}{ Without iodine-125 seed strands } \\
\hline & Pre-stenting & Post-stenting & Pre-stenting & Post-stenting \\
\hline Diameter of obstructed IVC segment, mm & $5 \pm 2$ & $2 \pm 3$ & $5 \pm 2$ & $2 \pm 3$ \\
\hline $\begin{array}{l}\text { Pressure difference between the distal } \\
\text { IVC obstruction and right atrium, } \mathrm{mmHg}\end{array}$ & $17 \pm 2$ & $6 \pm 2$ & $17 \pm 2$ & $6 \pm 2$ \\
\hline Score of obstruction & $2-3$ & $0-1$ & $2-3$ & $0-1$ \\
\hline $\begin{array}{l}\text { P-value for comparison within each } \\
\text { group, between pre- and post-stenting }\end{array}$ & $<0.05$ & & $<0.05$ & \\
\hline
\end{tabular}

IVC, inferior vena cava.
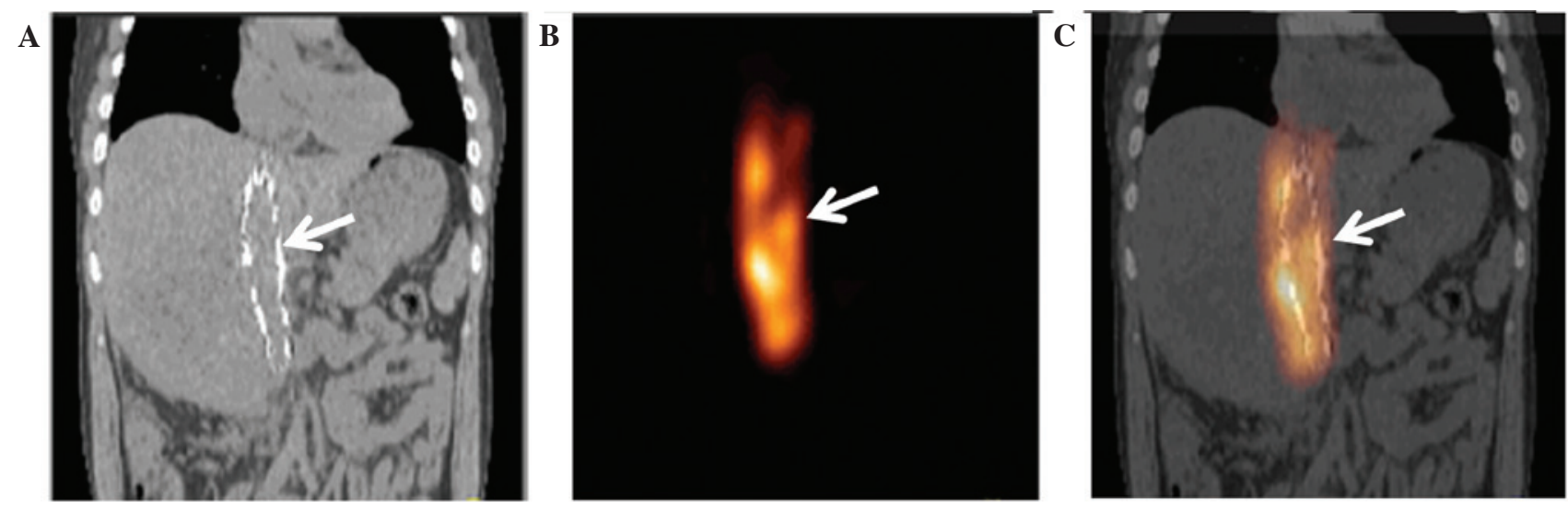

Figure 3. Sagittal plane SPECT/CT images of iodine-125 seed strand-containing IVC stents. (A) CT image of the stent (white arrow). (B) SPECT image of the iodine-125 seed strands (white arrow). (C) Merged A and B images. The white arrow indicates the cylinder-shaped radiation around the IVC stent. SPECT, single-photon emission computed tomography; CT, computed tomography; IVC, inferior vena cava.

$61 \pm 10$ years (50-75 years). Liver function grades in the study population were Child-Pugh A in 43 cases and Child-Pugh B in 9 cases. Clinical obstruction symptoms were 45 cases of mild edema, 7 cases of severe edema in the lower limbs, 8 cases of abdominal wall superficial varicosity and 2 cases of scrotal edema, while the average score of obstruction-associated symptoms prior to treatment was $2.4 \pm 0.5(2,3)$. Five patients suffered from IVC obstructions due to external pressure from retroperitoneal lymph node metastasis; 44 patients suffered from IVC obstructions due to the invasion of hepatic lesions; and in 3 patients the IVC obstructions were caused by tumor thrombus.

Tumor response rates. There was no CR case in either group. In the group treated with iodine-125 seed strands, there was $1 \mathrm{PR}, 16 \mathrm{SD}$ and $1 \mathrm{PD}$ cases; the tumor response rate was $94.4 \%$ (17/18). In the group without iodine-125 treatment, there were 2 PR, $10 \mathrm{SD}$ and $22 \mathrm{PD}$ cases; the tumor response rate was $35.3 \%$ (12/34). The tumor response rates were significantly different between the two groups $\left(\chi^{2}=16.6, \mathrm{P}<0.001\right)$.

Comparison of IVC obstructions between the two groups and within each group. In the iodine-125 seed strand group, all stents and iodine-125 seed strands were successfully implanted into the obstructed IVC segments, comprising a total of 18 stents and 20 iodine-125 seed strands ( 2 iodine- 125 seed strands for 2 patients, and 1 iodine-125 seed strand in the other 16 patients), containing 348 iodine-125 seeds, which was 16.7 \pm 6.9 (range, 10-33 seeds) for each patient in average. In the group without iodine-125 seed strands, all 34 stents were successfully implanted. The diameter of the obstructed IVC segments, the pressure difference between the distal IVC obstructed segment and the right atrium as well as the IVC obstruction scores did not significantly differ between the two groups; however, significant differences were found within each group, between pre-and post-stenting ( $\mathrm{P}<0.05$; Table II).

SPECT/CT scanning. At 1 day after the stenting, SPECT/CT images showed that implanted stents and iodine-125 seed strands were accurately located in the obstructed IVC segments of all patients without displacement. The radiation distribution of the iodine-125 seed strands in the stents was observed to be cylindrical with a radius of $\sim 1 \mathrm{~cm}$ (Fig. 3).

Complications. At 2 days after the procedure, acute renal failure occurred in 1 patient in the iodine-125 seed strand group with an increase in the serum creatinine concentration from $54 \mu \mathrm{mol} / 1$ prior to stenting to $637 \mu \mathrm{mol} / 1$ after stenting; however, the liver function and blood routine test results remained within the normal ranges and dialysis alleviated the 
symptoms. No serious complications occurred in the other patients.

\section{Discussion}

The anti-cancer effect of iodine-125 is thought to be based on the sustained release of 27.4-31.4 KeV X-rays and $35.5 \mathrm{KeV}$ $\gamma$-rays, which kill tumor cells via free oxygen radicals and ionization (9). The effective radiation radius of iodine-125 is only $17 \mathrm{~mm}$, which reduces the damaging effects on normal tissue and adjacent organs (10). Continuous low-dose radioactive iodine-125 induces the G2/M-phase arrest of tumor cells, which are then in turn also more sensitive to radiation $(11,12)$. In addition, implantation of iodine-125 leads to $\mathrm{CD}^{+}$and $\mathrm{CD}^{+}$cell activation, thereby producing antitumor immune responses (13). A randomized controlled study by Chen et al found that brachytherapy with iodine-125 following liver resection significantly prolonged the time to recurrence and overall survival rates (14). Nag et al observed high tumor complete or partial remission rates for unresectable hepatocellular carcinoma when using iodine-125 brachytherapy (15) and Chuan-Xing et al reported that it was effective and safe to perform chemoembolization and implantation of stents with iodine-125 seeds for patients with HCC and portal vein thrombus (16). In the present study, iodine-125 seeds were encapsulated into sterile plastic 4-F tubes and arranged in a line, thus creating iodine-125 seed strands, which were then placed between the stent and the vena cava wall. After release, the stent provided sufficient mechanical expansion force to fix the radioactive seed strands at a predetermined position. In addition, the continuous adherent effect of the stent (scaffolding) might reduce the incidence of lung metastasis and pulmonary embolism caused by tumor thrombus shedding, which is supported by the fact that no tumor thrombus shedding was observed in the present study. In this study, the tumor response rate of patients in the iodine-125 seed strand group was significantly better than that in the group without iodine-125 $(\mathrm{P}<0.001)$. In a previous study $(16)$, it was found that in cases of hepatocellular carcinoma with portal vein thrombosis, the intra-stent patency rates were 100,100 and $84.6 \%$ using iodine- 125 seeds vs. 100, 93.3 and $53.3 \%$ without iodine-125 seeds at 2 months, 6 months and 1 year, respectively, after stent deployment. Iodine-125 improved stent patencies; however for the difference in patency improvement to become evident, an extended period is necessary. In the present study, the average follow-up time of IVC obstruction was only $\sim 2$ months and a prospective study with a longer follow-up period is required to verify the effect of iodine-125 on intra-stent restenosis.

Previous studies have reported some complications associated with iodine-125, such as nausea, vomiting, and decreased white blood cell and platelet counts; however, the complications were relatively mild and spontaneous remission was common $(17,18)$. In the present study, acute renal failure occurred in 1 patient at 1 day after the procedure, but liver function and blood routine test results were normal. It is hypothesized that the complication was caused by TACE.

Although the present study has a small sample size and short follow-up time, it provides a new approach for IVC stent implantation using iodine-125 seeds. These preliminary experiences suggest that stents combined with iodine-125 seed strands are effective and safe for the treatment of HCC with IVC obstruction after chemoembolization. However, prospective and controlled studies with larger sample sizes and longer follow-up periods are required to verify its efficiency.

\section{References}

1. Parkin DM, Bray F, Ferlay J and Pisani P: Global cancer statistics, 2002. CA Cancer J Clin 55: 74-108, 2005.

2. Tang ZY, Ye SL, Liu YK, et al: A decade's studies on metastasis of hepatocellular carcinoma. J Cancer Res Clin Oncol 130: 187-196, 2004.

3. Kato Y, Tanaka N, Kobayashi K, Ikeda T, Hattori N and Nonomura A: Growth of hepatocellular carcinoma into the right atrium. Report of five cases. Ann Intern Med 99: 472-474, 1983.

4. Zeng ZC, Fan J, Tang ZY, et al: A comparison of treatment combinations with and without radiotherapy for hepatocellular carcinoma with portal vein and/or inferior vena cava tumor thrombus. Int J Radiat Oncol Biol Phys 61: 432-443, 2005.

5. Wu LL, Luo JJ, Yan ZP, et al: Comparative study of portal vein stent and TACE combined therapy with or without endovascular implantation of iodine-125 seeds strand for treating patients with hepatocellular carcinoma and main portal vein tumor thrombus. Zhonghua Gan Zang Bing Za Zhi 20: 915-919, 2012 (In Chinese).

6. Amols HI: Review of endovascular brachytherapy physics for prevention of restenosis. Cardiovasc Radiat Med 1: 64-71, 1999.

7. Sidawy AN, Weiswasser JM and Waksman R: Peripheral vascular brachytherapy. J Vasc Surg 35: 1041-1047, 2002.

8. Heysek RV: Modern brachytherapy for treatment of prostate cancer. Cancer Control 14: 238-243, 2007.

9. Lazarescu GR and Battista JJ: Analysis of the radiobiology of ytterbium-169 and iodine-125 permanent brachytherapy implants. Phys Med Biol 42: 1727-1736, 1997.

10. Nath R, Anderson LL, Luxton G, Weaver KA, Williamson JF and Meigooni AS: Dosimetry of interstitial brachytherapy sources: Recommendations of the AAPM Radiation Therapy Committee Task Group No. 43. Med Phys 22: 209-234, 1995

11. Strasser-Wozak EM, Hartmann BL, Geley S, et al: Irradiation induces $\mathrm{G} 2 / \mathrm{M}$ cell cycle arrest and apoptosis in p53-deficient lymphoblastic leukemia cells without affecting Bcl-2 and Bax expression. Cell Death Differ 5: 687-693, 1998.

12. Zhuang HQ, Wang JJ, Liao AY, Wang JD and Zhao Y: The biological effect of ${ }^{125} \mathrm{I}$ seed continuous low dose rate irradiation in CL187 cells. J Exp Clin Cancer Res 28: 12, 2009.

13. Xiang GA, Chen KY, Wang HN and Xiao JF: Immunological influence of iodine-125 implantation in patients with hepatocellular carcinoma resection. Nan Fang Yi Ke Da Xue Xue Bao 30: 292-294, 2010 (In Chinese).

14. Chen K, Xia Y, Wang H, Xiao F, Xiang G and Shen F: Adjuvant iodine-125 brachytherapy for hepatocellular carcinoma after complete hepatectomy: A randomized controlled trial. PLoS One 8: e57397, 2013.

15. Nag S, DeHaan M, Scruggs G, Mayr N and Martin EW: Long-term follow-up of patients of intrahepatic malignancies treated with iodine-125 brachytherapy. Int J Radiat Oncol Biol Phys 64: 736-744, 2006.

16. Chuan-Xing L, Xu H, Bao-Shan H, et al: Efficacy of therapy for hepatocellular carcinoma with portal vein tumor thrombus: Chemoembolization and stent combined with iodine-125 seed. Cancer Biol Ther 12: 865-871, 2011.

17. Armstrong JG, Anderson LL and Harrison LB: Treatment of liver metastases from colorectal cancer with radioactive implants. Cancer 73: 1800-1804, 1994.

18. Martinez-Monge R, Nag S, Nieroda CA and Martin EW: Iodine-125 brachytherapy in the treatment of colorectal adenocarcinoma metastatic to the liver. Cancer 85: 1218-1225, 1999. 